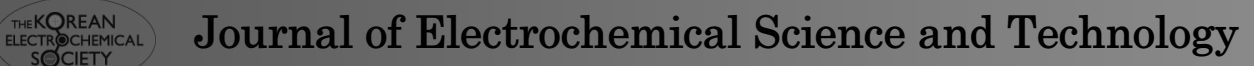

\title{
Electrosynthesis and Electrochemical Properties of Metal Oxide Nano Wire/ P-type Conductive Polymer Composite Film
}

\author{
S.O. Ranaei Siadat* \\ University of Shahid Beheshti, Tehran, Iran
}

\begin{abstract}
This study introduces a facile strategy to prepare metal oxide/conducting polymer nanocomposites that may have promising applications in energy storage devices. Ploy aniline/nano wire manganese dioxide $\left(\mathrm{PANI} / \mathrm{NwMnO} \mathrm{O}_{2}\right)$ was synthesized by cyclic voltammetry on glassy carbon electrode. Morphology and structure of the composite, pure PANI, $\mathrm{MnO}_{2}$ nanowires were fully characterized using XRD and SEM analysis. Electrochemical studies shows excellent synergistic effect between PANI and $\mathrm{MnO}_{2}$ nanowires which results in its capacitance increase and cycle stability against PANI electrode. Specific capacitances of PANI/NwMnO 2 and PANI were 456 and $190 \mathrm{~F} / \mathrm{g}$ respectively. The electrochemical performance of electrodes studied using cyclic voltammetry, Galvanostatic charge/discharge and impedance spectroscopy.
\end{abstract}

Keywors: super capacitors, nanowire, impedance, nanocomposite.

Received May 26, 2015 : Revised July 3, 2015 : Accepted July 6, 2015

\section{Introduction}

Electrochemical capacitors (ECs) represent an emerging class of energy storage devices that have attracted increasing attention because of a number of important features including high power density, fast charge/discharge rate, and excellent cycle stability $[1,2]$. In ECs, the electrode material is one of the extremely important factors affecting the performance of ECs (energy, power density, safety, cycle life, etc) $[3,4]$. Carbon-based materials [5], metal oxides [6], conductive polymers [7] and their composites $[8,9]$ have been used for producing ECs. High specific capacitance, flexibility and low cost are some advantages of conducting polymers, while they have disadvantages like shrinking nature during charge/discharge process. Based on its Mott-Schottky diagram, Polyaniline (PANI) is a p-type conductive polymer [10]. Because of excellent organic conductivity, biocompatibility and good environmental stability, PANI has been widely used in ECs and biosensors [11,12].

Many reports have been published on PANI and nano materials composites [13-17]. Increasing capacitance and stability is the goal of using PANI nano composite materials. There are two methods for PANI composite synthesis: chemical polymerization, and electropolymerization. In chemical method, the reaction product is always a powder. So, it has to be dried which leads to change the polymer structure $[18,19]$. Furthermore, in order to making electrode using the composite, binder is needed which in turn decreases its electrical and electrochemical performance.

*Corresponding author. Tel.:

E-mail address: Ranaeisiadat@hotmail.com

Open Access DOI: http://dx.doi.org/10.5229/JECST.2015.6.3.87

This is an Open Access article distributed under the terms of the Creative Commons Attribution Non-Commercial License (http://creativ ecommons.org/licenses/by-nc/3.0/) which permits unrestricted non-commercial use, distribution, and reproduction in any medium, provided the original work is properly cited. 
Electropolymerisation of PANI has many advantages: not needing oxidants, film uniformity on the electrode, saving the use of binder, decreasing contact resistance between polymer and current collector [20-22]. Because of synergistic effect between two composite components, Dramatic improvements have been achieved in electrochemical properties of composites.

$\mathrm{MnO}_{2}$ has been used as an active material in energy storage field. It has low cost, environmentally friendly nature, and various forms with different properties. So, It has been the subject of many researches as an electrode material for electrochemical capacitors [23-25]. In this paper we synthesized $\alpha-\mathrm{MnO}_{2}$ nano-wire which then was used to make $\mathrm{PANI} / \mathrm{NwMnO}_{2}$ in order to investigate the supercapacitive behavior and synergistic effect of these active materials together.

\section{Experimental}

\subsection{Chemicals}

All the chemical materials used in this work were Merck products by analytical grade and were used without further purification. Double distilled water was used throughout. Aniline (ANI) was doubly distilled and the resulting colorless liquid was kept in the dark at $5^{\circ} \mathrm{C} . \mathrm{MnO}_{2}$ nanowires were synthesized via a simple hydrothermal method. In a typical syn-

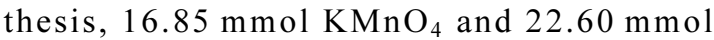
$\mathrm{MnSO}_{4} \cdot \mathrm{H}_{2} \mathrm{O}$ were dissolved in $120 \mathrm{~mL}$ deionized water under vigorous stirring. After $20 \mathrm{~min}$, the prepared mixture was transferred into a $125 \mathrm{~mL}$ Teflonlined stainless steel autoclave followed by heating at $140^{\circ} \mathrm{C}$ for $12 \mathrm{~h} \mathrm{[26].}$

\subsection{Synthesis of PANI and PANI/NwMnO ${ }_{2}$ com- posite electrodes}

$\mathrm{PANI} / \mathrm{NwMnO}_{2}$ composite was synthesized electrochemically by cyclic voltammetry in solution of $0.03 \mathrm{M}$ ANI in $\mathrm{H}_{2} \mathrm{SO}_{4} 1 \mathrm{M}, 0.2 \%$ wt. $\mathrm{MnO}_{2}$ nano wire and $0.005 \mathrm{M}$ of sodium dodecyl sulfate (SDS) that was dispersed in solution by sonication. PANI electrode was synthesized in same solution without $\mathrm{MnO}_{2}$ nano wire and SDS. Polymerizations were conducted by 10 cycles at the sweep rate of $50 \mathrm{mV} / \mathrm{s}$. The mass of PANI films was approximated assuming a current efficiency for the electro polymerization process of $100 \%$, using Faraday's law of electrolysis[27].

\subsection{Materials characterization and electrochemi- cal evaluation}

All electrochemical experiments were carried out by an Autolab General Purpose System PGSTAT 30 (Eco-chime, Netherlands). A conventional three electrode cell with an $\mathrm{Ag} / \mathrm{AgCl}$ reference electrode (Argental, $3 \mathrm{M} \mathrm{KCl}$ ) was used in order to electro polymerization of the PANI, the Platinum wire with a diameter of $0.5 \mathrm{~mm}$ and an exposed area of $0.65 \mathrm{~cm}^{2}$ was used as the counter electrode. A glassy carbon electrode with the area of $0.03 \mathrm{~cm}^{2}$ was used as the working electrode. A wide frequency range of $15 \mathrm{mHz}$ to $100 \mathrm{kHz}$ was used for EIS studies. Morphological investigations of the polymeric and composite films were carried out by using SEM (Philips XL 30). X-ray diffraction patterns were obtained from an X-ray diffrac-tometer (PANalytical X'PertPro) with a $\mathrm{Cu}-\mathrm{K}^{\alpha}$ monochromatized radiation source and a Ni filter.

\section{Results and Discussion}

Fig. 1(a, b, c) presents the SEM graphs of PANI/ $\mathrm{NwMnO}_{2}$ composite electrode. As can be seen, $\mathrm{MnO}_{2}$ nanowires have been coated on the surface of PANI filaments uniformly. The shape and structure of $\alpha-\mathrm{MnO}_{2}$ nanowire is shown in Fig. 1(d) It can be clearly seen that $\mathrm{MnO}_{2}$ nanowires is actually composed with uniform shape.

$\mathrm{X}$-ray diffraction was used to investigate the crystal structure of prepared materials. shows the XRD patterns of $\mathrm{MnO}_{2}$ nanowires, PANI and PANI/ $\mathrm{NwMnO}_{2}$ electrodes. For $\mathrm{MnO}_{2}$ nanowires, it can be seen clearly that all diffraction peaks can be exclusively indexed as the tetragonal $\alpha-\mathrm{MnO}_{2}$ (JCPDS 440141) and no other impurities were observed. XRD diagram of PANI/NwMnO 2 shows that by adding $\mathrm{MnO}_{2}$ nanowires to the PANI matrix, its crystal structure was changed and some peaks in XRD pattern of $\mathrm{PANI} / \mathrm{NwMnO}_{2}$ were appeared.

Cyclic voltammetry technique was used to evaluate the electrochemical performance of electrodes. Fig. 3(a) present's cyclic voltammograms of electropolymerised PANI and PANI/NwMnO 2 electrodes at $10^{\text {th }}$ cycle of electro polymerization process. It can be clearly seen that the electropolymerization charge of PANI/NwMnO 2 electrode is higher than that of PANI electrode and this has been considered as the contribution of faradic pseudo capacitance of 


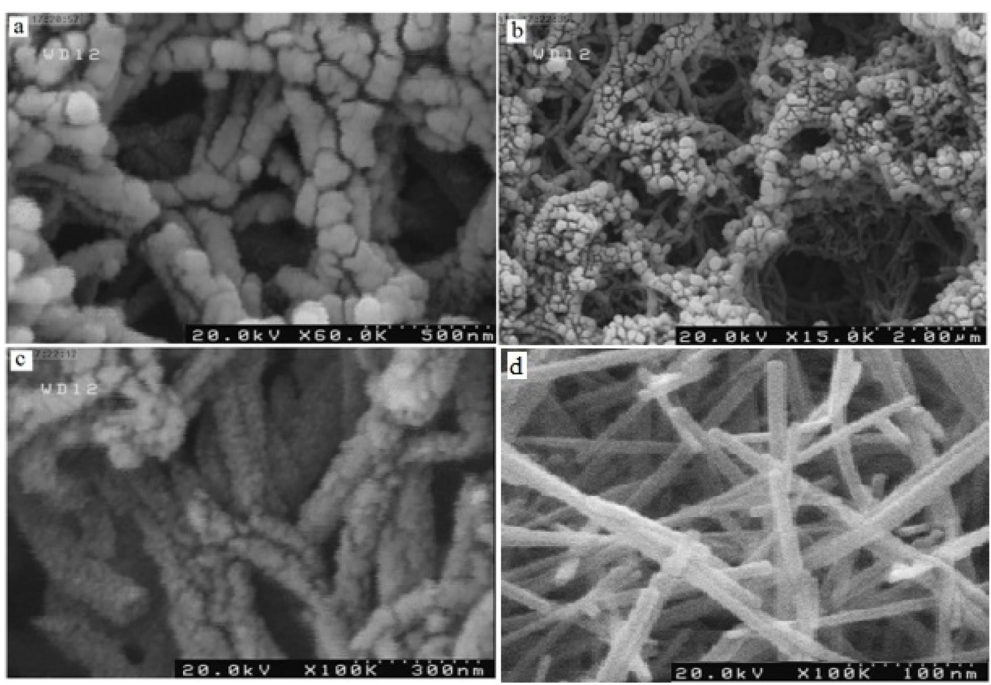

Fig. 1. SEM graphs of PANI /NwMnO $2(a, b, c)$ composite electrodes and $\mathrm{MnO}_{2}$ nano wire (d).

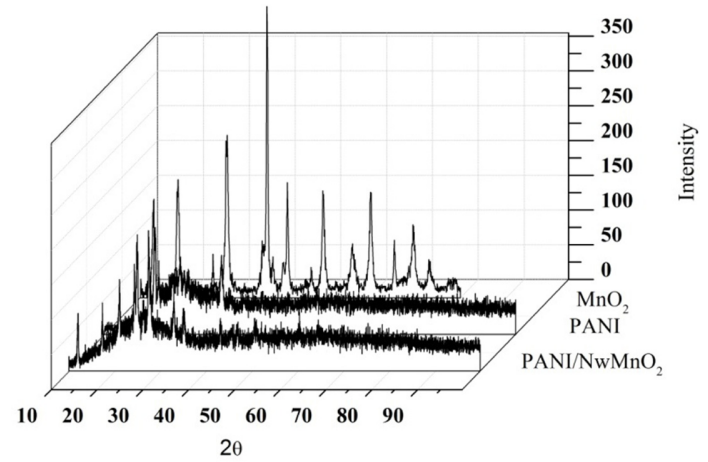

Fig. 2. XRD diagrams of $\mathrm{MnO}_{2}$ nanowire, PANI and PANI/ $\mathrm{NwMnO}_{2}$.

$\mathrm{MnO}_{2}$ nanowires and double layer behavior of PANI. In both cyclic voltammograms there are two redox peaks. The first one which is in negative potentials is related to the formation of free radicals in the polymerization of PANI and PANI/NwMnO 2 nano wire electrodes. The second one is due to oxidation of Polymer in both electrodes. Fig. 3(b) presents cyclic voltammograms of PANI and PANI/ $\mathrm{NwMnO}_{2}$ electrodes at the scan rate of $25 \mathrm{mV} / \mathrm{s}$ in $1 \mathrm{M} \mathrm{H}_{2} \mathrm{SO}_{4}$ solution. PANI/NwMnO 2 electrode, a remarkable difference can be seen in the cyclic voltammogram loop areas. This shows that the electrochemical behavior of the composite electrode is distinctly improved after adding $\mathrm{MnO}_{2}$ nanowires to PANI matrix. The charge storage in faradic process is achieved by electron transfer that leads to chemical changes in the electro active materials according to Faraday's law related to the potential [28,29]. Furthermore, symmetrical and rectangular shape of composite cyclic voltammogram curve shows an ideal capacitive behavior for this electrode involving two types of capacitive performances: contributing the electric double-layer capacitance produced by PANI and pseudo capacitive behavior of $\mathrm{MnO}_{2}$ nanowires. As shown in Fig. 3(b), a redox peak in potentials between $0.5-0.6 \mathrm{~V}$ were appeared that is attributed to PANI transition from emeraldine to pernigraniline form [30]. Specific capacitance (SC) of electrodes calculated from cyclic voltammogram curves according to following equation:

$$
C=\frac{1}{m v}
$$

Where $I$ is the current, $\mathrm{m}$ is the mass of reactive material and $i$ is the potential scan rate. The SC of PANI and PANI/ $\mathrm{MnO}_{2}$ electrodes at the scan rate of $25 \mathrm{mV} / \mathrm{s}$ were calculated 190 and $456 \mathrm{~F} / \mathrm{g}$ respectively.

Cyclic voltammograms of composite electrodes in $1 \mathrm{M} \mathrm{H}_{2} \mathrm{SO}_{4}$ media in different scan rates is shown in a. As can be seen the excellent capacitive performance of dPANI/NwMnO${ }_{2}$ electrode was also verified based on these curves. With an increase of scan rate the current response increases that indicate an ideally capacitive behavior of PANI/NwMnO${ }_{2}$ electrode [30]. Furthermore the good rectangular shape 

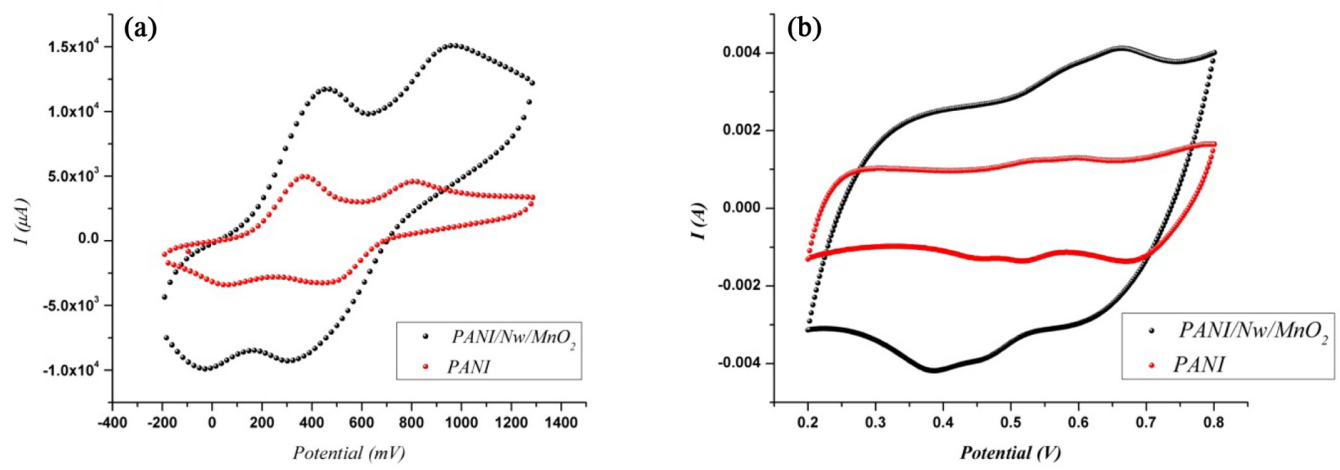

Fig. 3. (a) Comparative cyclic voltammograms for electropolymerization of PANI electrode and $\mathrm{PANI} / \mathrm{NwMnO}_{2}$ composite electrode at the 10th cyclicvoltamogram, (b) cyclic voltammogram curves of PANI and PANI/NwMnO${ }_{2}$ electrode at the scan rate of $25 \mathrm{mV} / \mathrm{s}$ in $1 \mathrm{M} \mathrm{H}_{2} \mathrm{SO}_{4}$ solution.
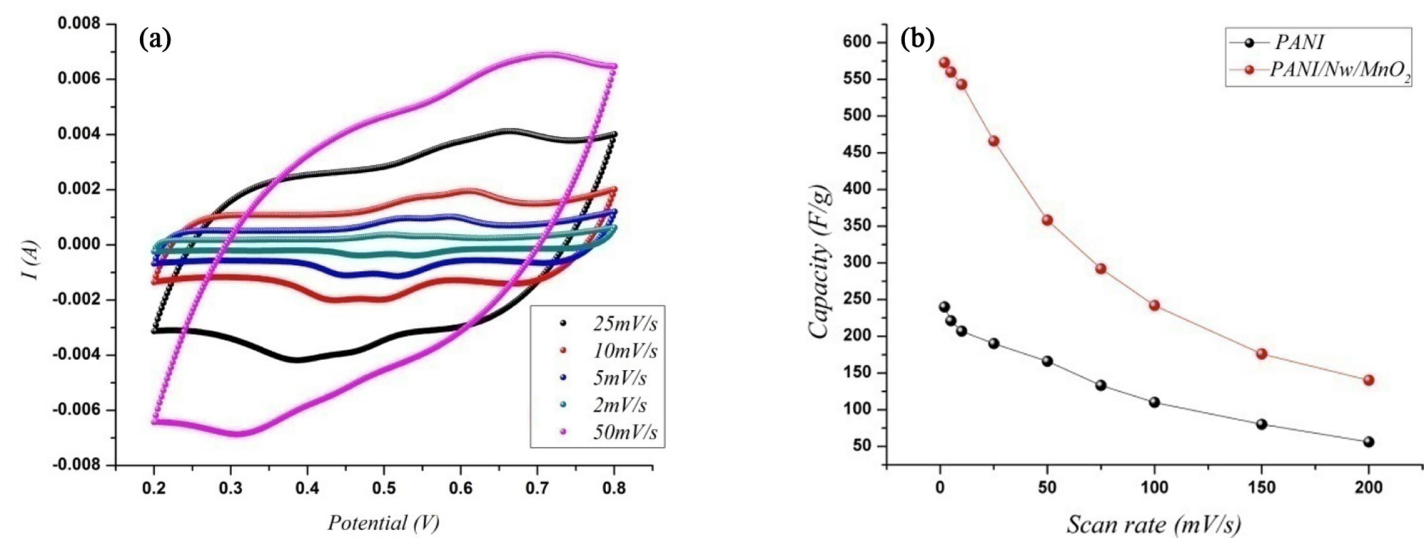

Fig. 4. (a) cyclic voltammograms of $\mathrm{PANI} / \mathrm{NwMnO}_{2}$ nanowires electrode at different scan rates in $1 \mathrm{M} \mathrm{H}_{2} \mathrm{SO}_{4}$ in the potential window of $0.2-0.8 \mathrm{~V}$, (b) variations of the specific capacitance for $\mathrm{PANI} / \mathrm{NwMnO}_{2}$ electrode as a function of the scan rate in $1 \mathrm{M} \mathrm{H} 2 \mathrm{SO} 4$ solution.

of PANI/NwMnO 2 voltammograms is remained up to the scan rate of $100 \mathrm{mV} / \mathrm{s}$. The deviation from rectangularity in cyclic voltammograms becomes obvious as scan rate increases. This phenomenon can be attributed to the electrolyte and film resistance, and this distortion is depending on scan rate. By increasing the sweep rate, active sites in electrode don't have enough time to react with ions.

Fig. 4(b) presents the calculated SCs of both electrodes at different scan rates. $\mathrm{PANI} / \mathrm{NwMnO}_{2}$ composite electrode shows SCs of 575 and $150 \mathrm{~F} / \mathrm{g}$ at the scan rates of 2 and $200 \mathrm{mV} / \mathrm{s}$, respectively, whereas at the same range these magnitudes for PANI electrode were calculated from 240 and $60 \mathrm{~F} / \mathrm{g}$. As can be seen, the capacitance of both electrodes decayed over the entire range of scan rate, but for $\mathrm{PANI} / \mathrm{NwMnO}_{2}$ electrode the slope of SC vs. scan rate was not only more than that of PANI electrode, but also less than it. This phenomenon can be resulted from the fact that both electrodes are porous and the presence of $\mathrm{MnO}_{2}$ nano wires do not cause to block the porosities of PANI film. Therefore, different electrochemical behaviors of the electrodes could come from the synergistic effect between $\mathrm{MnO}_{2}$ nanowires and PANI. Chemical methods of composite fabrication could motive to block some porosities of polymer film which cause to decay PANI super capacitive behavior. Therefore using electrochemical method, the whole pseudocapacitive behavior of PANI electrode could be saved.

Galvanostatic charge-discharge (CD) is one of the methods that have been used to study the capacitive 

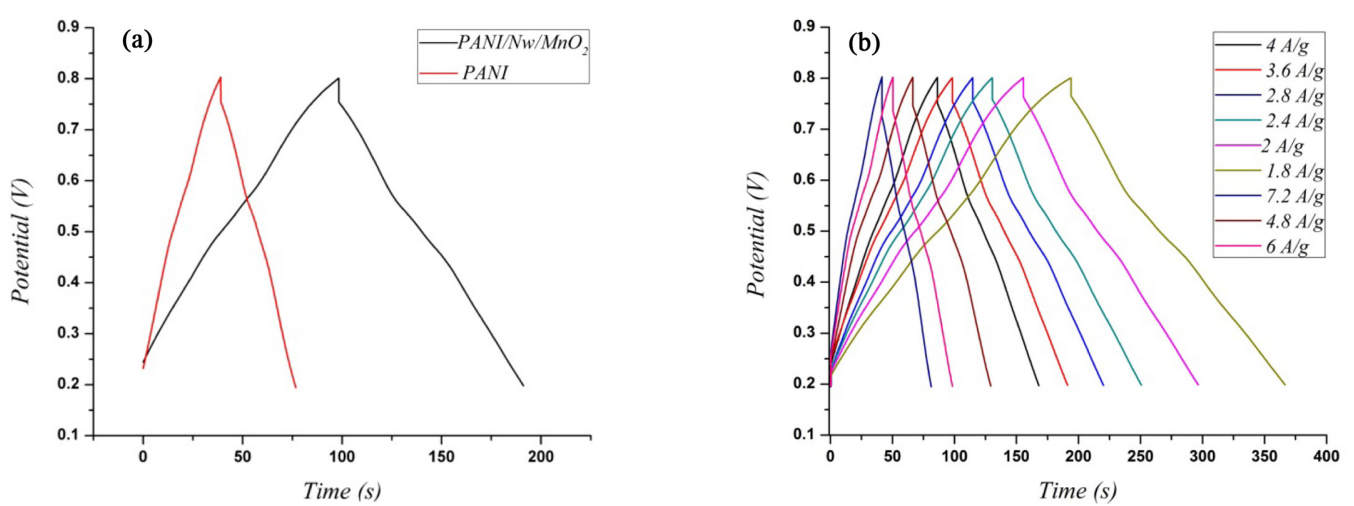

Fig. 5. (a) $\mathrm{CD}$ diagrams of PANI and $\mathrm{PANI} / \mathrm{NwMnO} 2$ nano wires electrodes at the current density of $3.6 \mathrm{~A} / \mathrm{g}$, (b) $\mathrm{CD}$ diagrams of PANI/MnO 2 electrode at $1.8,2,2.4,2.8,3.6,3.6,4,4.8,6$ and $7.2 \mathrm{~A} / \mathrm{g}$ in $1 \mathrm{M} \mathrm{H}_{2} \mathrm{SO}_{4}$ solution.

properties of active materials[31]. Fig. 5(a) shows the $\mathrm{CD}$ behavior of PANI and PANI/NwMnO 2 electrodes in the potential range from 0.2 to $0.8 \mathrm{~V}$ at the current density of $3.6 \mathrm{~A} / \mathrm{g}$. In this range, a triangular shape can be seen indicating that $\mathrm{PANI} / \mathrm{NwMnO}_{2}$ composite electrode have a good Columbic efficiency and ideal capacitive behavior in order to use for supercapacitors. The charge and discharge phase of PANI/NwMnO${ }_{2}$ lasts longer than that of PANI electrode which shows it has more capacitance than PANI. In addition, Because of increasing conductivity, using $\mathrm{MnO}_{2}$ nanowires cause to decrease the voltage drop (iR) which appeares in the curves during the change of current sign [31,32].

Fig. 5(b) presents the CD curves of PANI/ $\mathrm{NwMnO}_{2}$ electrode at various specific currents of 1.8, 2, 2.4, 2.8, 3.6, 3.6, 4, 4.8, 6 and 7.2 A/g. As can be seen, the shapes of all curves are approximate isosceles triangles which are the characteristic of super capacitors. Here, SC was measured according to the charge/discharge curves, using Eq. (2).

$$
\mathrm{SC}=\mathrm{I} /[-(\Delta \mathrm{E} / \Delta \mathrm{t}) \mathrm{m}]
$$

With I being the applied current; $-\Delta \mathrm{E} / \Delta \mathrm{t}$, the slope of discharge curve after voltage drop at the beginning of each discharge process (ESR); and $\mathrm{m}$ is the mass of composite electrodes. The highest SC for composite electrode was obtained when the current density for CD process was $1.8 \mathrm{~A} / \mathrm{g}$. The $\mathrm{SC}$ of PANI/ $\mathrm{NwMnO}_{2}$ changes in the range of 677 to $340 \mathrm{~F} / \mathrm{g}$. It can be seen that enhancing the specific current due to the intercalation of ions at the surface of active materials in the electrode/electrolyte interface, the specific capacitance values decrease. On the other hand, at low specific currents, the specific capacitance increases because there would be enough time for insertion and deinsertion of ions at deeper porosities of the active materials in the electrode/electrolyte interface.

EIS technique was used for further studying the supercapacitive behavior of polymeric film and composite electrodes. The Nyquist plots of PANI and $\mathrm{PANI} / \mathrm{NwMnO}_{2}$ electrodes is shown in a. As illustrated, there is a semicircle in high frequencies related to the charge transfer resistance $\left(\mathrm{R}_{c t}\right)$ caused by the Faradic Reactions and the double-layer capacitance $\left(\mathrm{C}_{d l}\right)$ at the contact interface between electrode and electrolyte solution. The magnitude of $\mathrm{R}_{\mathrm{ct}}$ in PANI/NwMnO ${ }_{2}$ electrode was smaller than that of PANI electrode, because the addition of $\mathrm{MnO}_{2}$ nanowires enhances the conductivity and improves charge transfer performance of $\mathrm{PANI} / \mathrm{NwMnO}_{2}$ electrode. Both electrodes exhibit a nearly linear branch in the low-frequency region. A transition to linearity at low frequencies exhibit an ideal capacitive behavior which was observed for $\mathrm{PANI} / \mathrm{NwMnO}_{2}$ electrode clearer than PANI one. The low frequency capacitance $\left(\mathrm{C}_{l f}\right)$ of each film was determined using Eq. (3):

$$
\mathrm{C}_{l f}=(2 \pi f \mathrm{Z} ")^{-1}
$$

where $f$ and Z" are the frequency and imaginary component of impedance respectively.

It can be seen that PANI/NwMnO 2 electrode has more capacitance than PANI one. These results also confirmed the data obtained by cyclic voltammetry and galvanostatic charge-discharge techniques. One 

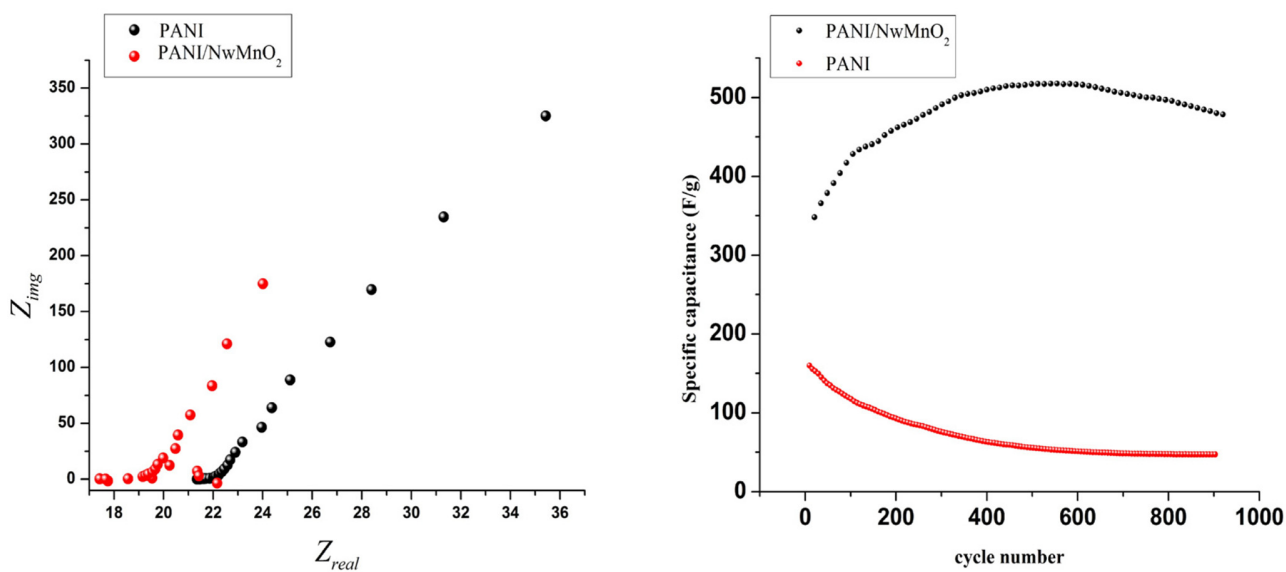

Fig. 6. Nyquist plots recorded from $10 \mathrm{kHz}$ to $0.01 \mathrm{~Hz}$ with an ac amplitude of $5 \mathrm{mV}$ for PANI and PANI/NwMnO${ }_{2}$ electrode, cycle ability of electrodes as a function of time during 20000 seconds at the scan rate of $50 \mathrm{mV} / \mathrm{s}$.

of the important features of supercapacitor electrodes is the stability of electrodes against consecutive or life cycles. Life cycles of both electrodes were investigated by cyclic voltametery at the scan rate of $50 \mathrm{mV} / \mathrm{s}$ for 900 cycles in $1 \mathrm{M} \mathrm{H}_{2} \mathrm{SO}_{4}$ solution (b). Synergistic effect of PANI and $\mathrm{MnO}_{2}$ nanowires is illustrated in the figure. As can be seen after 900 cycles, PANI electrode losses it's capacitance down to $70 \%$ while for $\mathrm{PANI} / \mathrm{NwMnO}_{2}$ electrode, the capacitance increases up to about $450^{\text {th }}$ cycle and then decreases with a gentle slope. Increasing the capacitance of composite electrode can be attributed to surface modification of $\mathrm{MnO}_{2}$ nano wires in consecutive cycles. During the polymerization of PANI/ $\mathrm{MnO}_{2}$ composite, some parts of $\mathrm{MnO}_{2}$ is covered by polymer chains which in turn are refreshed during next cycles resulted in increasing the electrode capacitance.

\section{Conclusion}

In summary, an electrochemical method to produce a polymer/metal oxide composite has been proposed. The morphology and the structure of the composite as well as pure PANI and $\mathrm{MnO}_{2}$ nanowires were fully characterized using XRD and SEM analysis. Electrochemical studies showed excellent synergistic effect between PANI and $\mathrm{MnO}_{2}$ nanowires resulted in increasing the capacitance and cycle stability of composite electrode against PANI one. Specific capacitance of $456 \mathrm{~F} / \mathrm{g}$ was obtained at the scan rate of $25 \mathrm{mV} / \mathrm{s}$ for composite electrode against $190 \mathrm{~F} / \mathrm{g}$ for PANI. Increasing the cycle ability of electrode is another advantage of using $\mathrm{MnO}_{2}$ nanowires polymer matrix.

\section{Acknowledgements}

The authors would like to express their deep gratitude to the Iranian Nano Council and TAHA Biotech company for supporting this work.

\section{References}

[1] Hall, P.J., et al., Energy storage in electrochemical capacitors: designing functional materials to improve performance. Energy \& Environmental Science, 2010. 3(9): p. 1238-1251.

[2] Simon, P. and Y. Gogotsi, Materials for electrochemical capacitors. Nature materials, 2008. 7(11): p. 845-854.

[3] Xia, K., et al., Hierarchical porous carbons with controlled micropores and mesopores for supercapacitor electrode materials. Carbon, 2008. 46(13): p. 1718-1726.

[4] Wu, F. and B. Xu, Progress on the application of carbon nanotubes in supercapacitors. New Carbon Materials, 2006. 21(2): p. 176-184.

[5] Zhao, D., et al., An electrochemical capacitor electrode based on porous carbon spheres hybrided with polyaniline and nanoscale ruthenium oxide. ACS applied materials \& interfaces, 2012. 4(10): p. 55835589.

[6] Sun, Z. and X. Lu, A solid-state reaction route to anchoring $\mathrm{Ni}(\mathrm{OH}) 2$ nanoparticles on reduced graphene oxide sheets for supercapacitors. Industrial \& Engineering Chemistry Research, 2012. 51(30): p. 99739979.

[7] Lefebvre, M., et al., Chemical synthesis, characterization, and electrochemical studies of poly (3, 4-ethylenedioxy- 
thiophene)/poly (styrene-4-sulfonate) composites. Chemistry of materials, 1999. 11(2): p. 262-268.

[8] Xu, D., et al., Fabrication of free-standing hierarchical carbon nanofiber/graphene oxide/polyaniline films for supercapacitors. ACS applied materials \& interfaces, 2013. 6(1): p. 200-209.

[9] Zhang, X., et al., Investigation of a Branchlike MoO3/ Polypyrrole Hybrid with Enhanced Electrochemical Performance Used as an Electrode in Supercapacitors. ACS applied materials \& interfaces, 2014. 6(2): p. 11251130.

[10] Ehsani, A., M. Mahjani, and M. Jafarian, An electrochemical study of the synthesis and properties of multi-walled carbon nanotube/poly ortho aminophenol composites. Synthetic Metals, 2011. 161(15): p. 17601765.

[11] Lei, Z., Z. Chen, and X. Zhao, Growth of polyaniline on hollow carbon spheres for enhancing electrocapacitance. The Journal of Physical Chemistry C, 2010. 114(46): p. 19867-19874.

[12] Dhand, C., et al., Recent advances in polyaniline based biosensors. Biosensors and Bioelectronics, 2011. 26(6): p. 2811-2821.

[13] Patil, A., et al., Issue and challenges facing rechargeable thin film lithium batteries. Materials research bulletin, 2008. 43(8): p. 1913-1942.

[14] de Heer, W.A., et al., Electron field emitters based on carbon nanotube films. Advanced Materials, 1997. 9(1): p. 87-89.

[15] Rao, C.N.R., et al., Nanotubes. ChemPhysChem, 2001. 2(2): p. 78-105.

[16] Liu, Z., et al., Organizing single-walled carbon nanotubes on gold using a wet chemical self-assembling technique. Langmuir, 2000. 16(8): p. 3569-3573.

[17] Shabani Shayeh, J., P. Norouzi, and M.R. Ganjali, Studying the supercapacitive behavior of a polyanilinel nano-structural manganese dioxide composite using fast Fourier transform continuous cyclic voltammetry. RSC Advances, 2015. 5(26): p. 20446-20452.

[18] Ates, M., Review study of electrochemical impedance spectroscopy and equivalent electrical circuits of conducting polymers on carbon surfaces. Progress in Organic Coatings, 2011. 71(1): p. 1-10.

[19] Jurewicz, K., et al., Supercapacitors from nanotubes/ polypyrrole composites. Chemical Physics Letters, 2001. 347(1): p. 36-40.

[20] Molina, J., et al., Electrochemical polymerisation of aniline on conducting textiles of polyester covered with
polypyrrole/AQSA. European Polymer Journal, 2009. 45(4): p. 1302-1315.

[21] Ehsani, A., M. Mahjani, and M. Jafarian, Electrosynthesis of poly ortho aminophenol films and nanoparticles: A comparative study. Synthetic Metals, 2012. 162(1): p. 199-204.

[22] Ehsani, A., et al., Poly ortho aminophenol/TiO 2 nanocomposite: electrosynthesis and characterization. Synthetic Metals, 2013. 165: p. 51-55.

[23] Long, J.W., A.L. Young, and D.R. Rolison, Spectroelectrochemical characterization of nanostructured, mesoporous manganese oxide in aqueous electrolytes. Journal of the Electrochemical Society, 2003. 150(9): p. A1161-A1165.

[24] Reddy, R.N. and R.G. Reddy, Synthesis and electrochemical characterization of amorphous $\mathrm{MnO} 2$ electrochemical capacitor electrode material. Journal of Power Sources, 2004. 132(1): p. 315-320.

[25] Hsieh, Y.-C., et al., Investigation on capacity fading of aqueous $\mathrm{MnO} 2 \cdot \mathrm{nH} 2 \mathrm{O}$ electrochemical capacitor. Journal of Power Sources, 2008. 177(2): p. 660-664.

[26] Sadeghinia, M., M. Rezaei, and E. Amini, Preparation of $\dot{a}-\mathrm{MnO} 2$ nanowires and its application in low temperature $\mathrm{CO}$ oxidation. Korean Journal of Chemical Engineering, 2013. 30(11): p. 2012-2016.

[27] Plesu, N., et al., Effect of temperature on the electrochemical synthesis and properties of polyaniline films. Journal of Non-Crystalline Solids, 2010. 356(20): p. 1081-1088.

[28] Conway, B.E., Electrochemical supercapacitors: scientific fundamentals and technological applications. 2013: Springer Science \& Business Media.

[29] Li, X., et al., In-situ polymerization of polyaniline on the surface of graphene oxide for high electrochemical capacitance. Thin Solid Films, 2015. 584: p. 348-352.

[30] Wang, H., et al., Effect of graphene oxide on the properties of its composite with polyaniline. ACS applied materials \& interfaces, 2010. 2(3): p. 821-828.

[31] Nam, K.-W., et al., Pseudocapacitive properties of electrochemically prepared nickel oxides on 3dimensional carbon nanotube film substrates. Journal of Power Sources, 2008. 182(2): p. 642-652.

[32] Hulicova-Jurcakova, D., et al., Graphitic carbon nanofibers synthesized by the chemical vapor deposition (CVD) method and their electrochemical performances in supercapacitors. Energy \& Fuels, 2008. 22(6): p. 4139-4145. 